\title{
CADASIL: A HEREDITARY CEREBROVASCULAR DISEASE AS MODEL FOR COMMON VASCULAR ISCHEMIC DEMENTIA
}

\section{Maria Teresa Dotti}

\section{Department of Neurological Sciences and Behaviour University of Siena}

Together with Alzheimer's disease, vascular dementia (VD) is the most frequent cause of progressive cognitive deterioration. The most common form of VD, subcortical vascular ischemic disease of the brain (SVIDB), affects elderly subjects who generally have a medical history of common vascular risk factors, especially hypertension. The clinical picture is generally progressive or stepwise due to recurrent stroke, and besides cognitive deterioration includes depression and problems with walking, swallowing and sphincter control. Magnetic resonance imaging (MRI), which is crucial in the diagnosis and follow-up of SVIDB, shows small subcortical infarcts (ischemic lacunae) and diffuse vascular damage in the white matter (vascular leucoencephalopathy).

A genetic cause of VD was recently described and characterised at molecular level. Cerebral autosomal dominant arteriopathy with subcortical infarcts and leukoencephalopathy (CADASIL) (Joutel et al. 1996) manifests clinically with migraine with aura, recurrent stroke/TIA(transistory ischemic attack) and progressive cognitive deterioration and is an ideal monogenic model of common VD (Kalimo 2002). The cause of ischemic brain damage in CADASIL is microangiopathy with deposition of electondense material (granular osmiophilic material, GOM) in the basement membrane.

Although clinical expression of CADASIL is typically brain-related, ultrastructural evidence of GOM deposits is also found in skin vessels and is of diagnostic value. Though $100 \%$ specific, the sensitivity of this evidence as a diagnostic screening method is controversial and was recently demonstrated by our group to be below 50\% (Malandrini et al. 2007).

The functional consequences of vascular alterations in the pathogenesis of CADASIL are still unclear. The prevalent hypothesis is that progressive damage to vascular smooth muscle cells (VSMCs) impairs selfregulation of brain vessels and progressively reduces brain perfusion. The molecular defect in CADASIL was identified as a mutation on the Notch 3 gene
(Tournier-Lasserve et al. 1993). The gene codes for the Notch3 transmembrane receptor which is essential for cell differentiation and signalling during development. In adults, the function of this receptor is unknown, but it may be involved in inhibition of apoptosis, favouring VSMC survival (Wang et al. 2002). About a hundred different mutations have been described (Federico et al. 2005), more than 70\% on exons 3 and 4. A recent genetic study of the first Italian families (Dotti et al. 2005) showed a peculiar mutational profile with a very low frequency of hot spot mutations on exons 3 and 4 and different distributions of mutations in relation to geographic origin. Since 2001, our group has identified 35 CADASIL familes from different geographical areas with more than 100 affected subjects (Dotti et al. 2005). All pathogenic mutations were on exons 2-24 that code one of 34 epidermal growth factor (EGF)-like repeat domains in the extracellular part of the Notch transmembrane receptor. A series of mutations, most of which do not involve amino acid substitutions (unpublished data), was also found; the roles of these mutations is still unclear.

Fig.1:

Notch3 gene mutation distribution in different exons

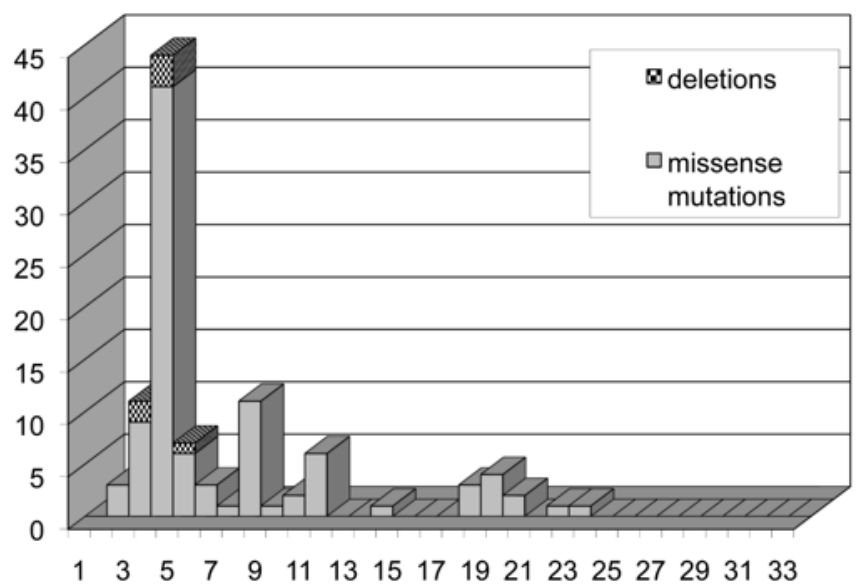


The few studies on genotype-phenotype relations have shown quite weak correlations (Singhal et al. 2004), suggesting that other genetic and/or environmental factors modulate clinical expression, which is highly variable, even in the same family. Common vascular risk factors do not have a causal role in CADASIL, however their possible influence on the expression of clinical phenotype is an interesting problem investigated by our group through studies that monitored systemic arterial pressure and heart rate over 24 hours (Rufa et al. 2005, 2007).

Besides the classical clinical manifestations (migraine, TIA/stroke, dementia and psychological disorders), CADASIL patients have been reported to have atypical brain-related and extraneurological symptoms, including eye alterations (Rufa et al. 2004 a, b, 2005, 2008) and heart alterations with a high prevalence of rightto-left shunts detected by transcranial doppler sonography (Zicari et al. 2008).

Magnetic resonance imaging is crucial for diagnostic screening for leukoencephalopathies. In CADASIL, diffuse and focal changes in white matter are fairly aspecific with respect to those found in common forms of vascular and non vascular leukoencephalopathies, such as multiple sclerosis and undefined late-onset leukodystrophy. As in the common, classic, age-related form of VD, MRI shows diffuse subcortical leukoencephalopathy of variable severity and lacunar infarcts of basal ganglia and pons. MRI findings are on the whole very similar to those observed in other forms of age-related leukoencephalopathy and demyelinating forms such as multiple sclerosis (MS) and late-onset leukodystrophy and no distinctive radiological criteria have yet been identified (van den Boom 2003). The relation between characteristics, MRI evidence of lesion severity and clinical manifestations is also controversial. Our MRI study of asymptomatic carriers of the Notch3 mutation, which not only have well-known early white matter changes but also quantitative changes that are an expression of early involvement of the cerebral cortex as well, aroused considerable interest (Stromillo et al. 2009).

Fig.2: Brain MRI in CADASIL in severe (a) and mildly (b) affected subjects

(a)

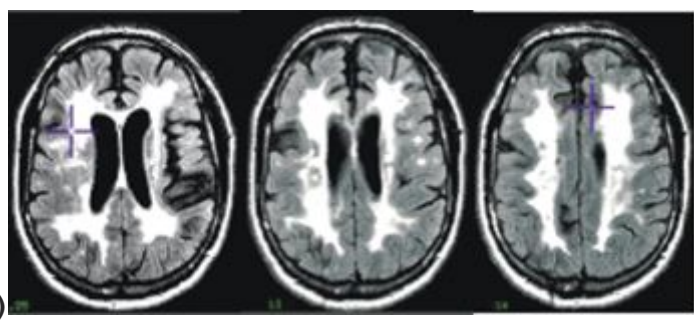

(b)

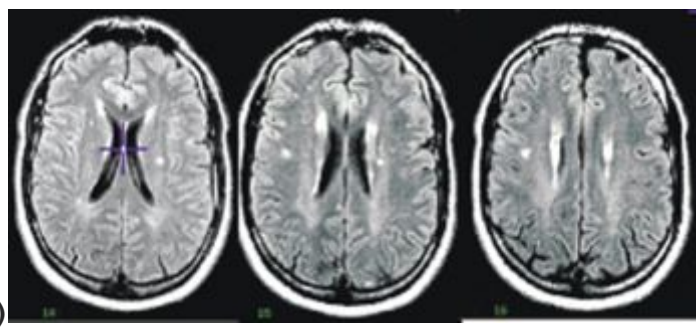

There is currently no specific therapy for this disease. Anticlotting agents are recommended. A recent multicentric study tested the efficacy, safety and tolerability of donezepil in CADASIL patients with cognitive deterioration.

\section{REFERENCES}

1. Dotti MT, Federico A, Mazzei $R$ et al. The spectrum of Notch 3 mutations in 28 Italian CADASIL families. JNNP 2005,76:763-8

2. Federico A, Bianchi S, Dotti MT. The spectrum of mutations for CADASIL diagnosis. Neurol Sci 2005,26:117-124 3. Kalimo $\mathrm{H}$ et al. CADASIL: a common form of hereditary arteriopathy causing brain infarcts and dementia. Brain Pathol 2002;12:371-384

4. Joutel A et al. Notch 3 mutations in CADASIL, a hereditary adult-onset condition causing stroke and dementia. Nature 1996; 383: 707-710

5. Malandrini A, Gaudiano C. Dotti MT et al. Diagnostic value of ultrastructural skin biopsy studies in CADASIL. Neurology 2007; 68: 1430-2

6. Rufa A, De Stefano N, Dotti MT et al. Acute unilateral visual loss as first symptom of cerebral autosomal dominant arteriopathy with subcortical infarcts and leukoencephalopathy. Arch Neurol 2004, 61:577-580a

7. Rufa A, Dotti MT, Franchi M et al. Systemic blood pressure profile in cerebral autosomal dominant arteriopathy with subcortical infarcts and leukoencephalopathy (CADASIL). Stroke 2005, 36:2554-8

8. Rufa A, Guideri F, Acampa M, Cevenini G, Stromillo $M L$, De Stefano N, Federico A, Dotti MT Cardiac autonomic nervous system and risk of arrhythmias in cerebral autosomal dominant arteriopathy with subcortical infarcts and leukoencephalopathy (CADASIL). Stroke 2007;38:276-280 9. Rufa A, Cerase A, Monti L, Battisti C, Forte F, Federico A, Dotti MT Acute vestibular syndrome in a patient with cerebral autosomal dominant leukoencephalopathy with subcortical infarcts and leukoencephalopathy (CADASIL). J Neurol Sci 2008

10. Singhal S, Bevan S, Barrick et al. The influence of genetic and cardiovascular risk factors on the CADASIL phenotype. Brain 2004; 127:2031-2038

11. Stromillo ML, Dotti MT et al. Focal and diffuse tissue damage in pre-symptomatic CADASIL. Neurol Sci 2007, vol 28 supplement, $p 280$

12. Tournier-Lasserve E et al. Cerebral autosomal dominant arteriopathy with subcortical infarcts and leukoencephalopathy maps on chromosome 19q12. Nat Genet 1993; 3, 256-259

13. Van den Boom R, Lesnik Oberstein SAJ, Ferrari MD et al. Cerebral autosomal dominant arteriopathy with subcortical infarcts and leukoencephalopathy: MR imaging findings at different ages - 3rd-6th decades. Radiology. 2003;229:683-90

14. Wang W, Prince CZ, Mou Y, Pollman MJ. Notch3 signaling in vascular smooth muscle cells induces c-FLIP expression via ERK/MAPK activation. Resistance to Fas ligand-induced apoptosis. J Biochem Chem 2002;277:21 7239

15. Zicari E, Tassi R, Dotti MT et al. Right-to-left shunt in CADASIL patients - prevalence and correlation with clinical and MRI findings. Stroke 2008,39:2155-7 